\title{
Measuring Energy, Estimating Hamiltonians, and the Time-Energy Uncertainty Relation
}

\author{
Y. Aharonov \\ School of Physics and Astronomy, Tel Aviv University, Tel Aviv 69978, Israel.f \\ S. Massar \\ Service de Physique Théorique, Université Libre de Bruxelles, \\ C.P. 225, Bvd. du Triomphe, B1050 Bruxelles, Belgium. \\ S. Popescu \\ H.H. Wills Physics Laboratory, University of Bristol, Tyndall Avenue, Bristol BS8-1TL, U.K.円
}

(Dated: October 24, 2018)

\begin{abstract}
Suppose that the Hamiltonian acting on a quantum system is unknown and one wants to determine what is the Hamiltonian. We show that in general this requires a time $\Delta t$ which obeys the uncertainty relation $\Delta t \Delta H \gtrsim 1$ where $\Delta H$ is a measure of how accurately the unknown Hamiltonian must be estimated. We apply this result to the problem of measuring the energy of an unknown quantum state. It has been previously shown that if the Hamiltonian is known, then the energy can in principle be measured with arbitrarily large precision in an arbitrarily short time. On the other hand we show that if the Hamiltonian is not known then an energy measurement necessarily takes a minimum time $\Delta t$ which obeys the uncertainty relation $\Delta t \Delta E \gtrsim 1$ where $\Delta E$ is the precision of the energy measurement. Several examples are studied to address the question of whether it is possible to saturate these uncertainty relations. Their interpretation is discussed in detail.
\end{abstract}

PACS numbers: 03.65.Ta

\section{INTRODUCTION}

The uncertainty relations play a central role in quantum mechanics. Their importance lies in the fact that they express in a succinct manner the fundamental limitations on measurements imposed by quantum mechanics. In particular it is these limitations that guarantee that the mathematical formalism of the theory is free from contradictions with experiment.

The time-energy uncertainty however has a particular status because time is an external parameter in the theory and the energy operator plays a special role since it determines the temporal evolution. Thus the time energy uncertainty does not follow from the commutation relations of two operators, but is determined indirectly, for instance from the mathematical properties of the Fourier transform with respect to the time variable. The interpretation and status of the time-energy uncertainty should therefore be examined with particular care.

In this paper we shall concentrate on energy measurements. By analogy with other measurements, one expects that the time-energy uncertainty expresses a fundamental constraint energy measurements must satisfy. The aim of this paper is to clarify whether such a constraint exists, and what should be its interpretation.

\footnotetext{
*Also at Departement of Physics, University of South Carolina, Columbia, SC 29208.

${ }^{\dagger}$ Also at BRIMS, Hewlett-Packard Labs., Stoke Gifford, Bristol BS12-6QZ, U.K.
}

Suppose that one must measure the energy of an unknown quantum state. One's first intuition[1] in this case is that that the energy of an unknown state can be determined to accuracy $\Delta E$ only if the duration $\Delta t$ of the measurement is larger than $1 / \Delta E$ (since $\Delta t \Delta E \geq 1$ ), where we set $\hbar=1$. Here and throughout, by accuracy of the measurement, we mean how much the result of the measurement differs from the result of an ideal von Neumann measurement of the Hamiltonian operator $H$. This will be defined with precision below.

Surprisingly, this intuition is incorrect. It is possible to measure the energy of an unknown quantum state to arbitrarily high accuracy in an arbitrarily short time [3]. As an illustration consider a spin $1 / 2$ particle with a magnetic moment $\mu$ in a magnetic field $\vec{B}_{0}=B_{0} \overrightarrow{1}_{z}$ pointing along the $z$ direction. In order to measure the energy of the spin, one can apply a strong magnetic field $\vec{B}(z)=B(z) \overrightarrow{1}_{z}$ pointing in the $z$ direction with a gradient in the $z$ direction. This realizes a Stern-Gerlach measurement of $\sigma_{z}$, hence of the energy of the particle. The time necessary for this measurement depends on the magnitude of the additional magnetic field $B(z)$, not on the original magnetic field $B_{0}$. Since $B(z)$ can be arbitrarily large, the energy can be determined in an arbitrarily short time. Another example, which is discussed in detail in the original article, is the measurement of the energy $H=p^{2} / 2 m$ of a free particle.

Thus the time-energy uncertainty seems not to apply to energy measurements. Is this result universal? Or are there cases where the time-energy uncertainty does apply and measuring energy to an accuracy $\Delta E$ does require a time $\Delta t$ limited by $\Delta t \Delta E \geq 1$ ? 
We first note that if one attempts to devise an energy measurement, it is often the case that this measurement will take a time that satisfies the time-energy uncertainty. We refer for instance to the example considered by Landau and Peirels [1], see also Landau and Lifshitz[2], in which the kinetic energy of a particle is measured by letting it collide with another particle initially at rest. In this example if the energy is to be measured with precision, the initial momentum of the particle at rest must be well known, but then its position is spread out and the time at which the measurement takes place is uncertain. Another well known example is the radiative decay of an excited state. The emission of a photon by an excited atom that decays to its ground state can be viewed as a measurement of the energy of the electronic state of the atom. The mean time it takes for the photon to be emitted, that is the lifetime of the excited state, is then interpreted as the mean time it takes to make the measurement. This lifetime is related to the line width, that is to the uncertainty of the energy measurement, by the time-energy uncertainty. Following [3] one would argue that such energy measurements, which satisfy the time-energy uncertainty, are simply badly designed, and that one can in principle make energy measurements that do not obey the time-energy uncertainty. Nevertheless it is surprising that so many energy measurements do obey the time-energy uncertainty. We would like to know what makes some energy measurements less efficient than others.

There are also some particular circumstances in which the time-energy uncertainty must be obeyed in an energy measurement. Specifically consider the situation in which one wants to measure the energy of an isolated system. Since it is isolated, ie. uncoupled to any exterior degrees of freedom, the measuring apparatus must be internal to the system itself. In [4] it is argued that this constraint implies that measuring the total energy of an isolated system to accuracy $\Delta E$ requires a time $\Delta t$ that satisfies $\Delta t \Delta E \geq 1$. Contrary to the examples mentioned in the preceeding paragraph, where a more sophisticated strategy could in principle measure the energy in a smaller time than that given by the uncertainty relation, in this case the uncertainty relation must be obeyed.

Thus the present status of the time-energy uncertainty in the context of energy measurements is unsatisfactory. Different examples suggest conflicting interpretations.

As we shall prove bellow, the resolution of this puzzle is the following. When the Hamiltonian of the system is known the conclusions of [3] hold, namely we can measure the energy as precisely as we want in a time as short as we want. On the other hand, whenever the Hamiltonian of a system is completely unknown, determining what is the Hamiltonian to precision $\Delta H$ requires a time $\Delta t$ given by $\Delta t \Delta H \geq 1$.

The origin of the conflicting interpretations of the timeenergy uncertainty relation in the context of estimating the energy of an unknown state is now clear. In some cases the energy measurement can also serve to estimate an unknown Hamiltonian. In these cases, because the measurement can serve this dual purpose, it requires a time $\Delta t$ which is limited by the accuracy with which it could estimate an unknown Hamiltonian. On the other hand the measurements envisaged in [3] cannot estimate an unknown Hamiltonian. These measurements measure an operator $A$ ( $\sigma_{z}$ in the example above, or the momentum $p$ in the example of [3]). If the Hamiltonian is a function of this operator $H=f(A)$ (for instance $H=\mu B_{0} \sigma_{z}$ or $\left.H=p^{2} / 2 m\right)$, then the measurement of $A$ can be used to determine the energy of an unknown state. Such a von Neumann measurement of an operator $A$ can be realized in an arbitrarily short time. But if the time is very short, the measurement is brutal, that is the interaction between the measuring apparatus and the system dominates the evolution. It is therefore impossible to use such a measurement to estimate an unknown Hamiltonian since the action of the original Hamiltonian is completely masked by the interaction.

Therefore in order to understand the interpretation of the time-energy uncertainty relation in the context of energy measurements, it is mandatory to first understand the limitations quantum mechanics imposes on the estimation of the Hamiltonian acting on a system when the Hamiltonian is unknown. This problem is in a certain sense the dual of the standard problem of quantum information in which one must estimate the state of a quantum system. Here one must estimate the dynamics. This is a fundamental problem in quantum mechanics. In fact many experimental situations can be phrased in this language. For instance there could be an unknown force acting on a particle which one wants to estimate. Or the Hamiltonian could depend on an unknown parameter which one wants to estimate.

We show in the present paper that the precision with which one can estimate the dynamics and the time required for this estimation are related by an uncertainty relation

$$
\Delta t \Delta H \geq 1
$$

where the precise meaning of $\Delta H$ depends on the details of the problem.

It is intuitively obvious that the dynamics of a quantum system cannot be estimated instantaneously. Indeed suppose that initially the state is $\psi_{0}$. Then after a time $t$ the state has evolved to $\psi(t, H)=e^{-i t H} \psi_{0} \simeq \psi_{0}+i t H \psi_{0}$. From this expression it is clear that in order to estimate the dynamics, ie. to estimate $H$, sufficient time must elapse that the zero'th order term $\psi_{0}$ in the expansion in $t$ does not dominate. Where this the case, the states $\psi(t, H)$ would be almost identical to $\psi_{0}$ and hence undistinguishable. The results reported in this paper make this qualitative statement precise.

The problem of estimating the dynamics has been considered recently by Childs, Preskill and Renes [5]. Their results constitute a starting point for our discussion.

The remainder of the article is organized as follows. We first discuss the problem of distinguishing with cer- 
tainty between two Hamiltonians. Next we consider the case where one only wants to estimate with finite error probability which of the two Hamiltonians is the true Hamiltonian. Then we turn to the problem of estimating what is the Hamiltonian when one has no prior knowledge about the Hamiltonian. Finally we go back to the problem of estimating the energy of an unknown state. We discuss how it is related to estimating an unknown Hamiltonian. We prove that if the Hamiltonian is completely unknown then the time necessary to estimate the energy and the precision with which the energy is estimated must obey a time-energy uncertainty relation. We illustrate the problem of estimating the energy of a state when the Hamiltonian is completely or partially unknown by several examples.

\section{DISTINGUISHING BETWEEN TWO HAMILTONIANS}

\section{A. Minimum time necessary to distinguish between two unknown Hamiltonians}

In this section we consider the problem of estimating the Hamiltonian acting on a system when the Hamiltonian is unknown. We consider in this section the special case in which there are only two possible Hamiltonians that could act on the system, $H_{1}$ or $H_{2}$, and one must distinguish with certainty which it is.

This particular problem has been considered previously by Childs, Preskill and Renes [5]. They show that the minimum time $\Delta t$ to determine with certainty the Hamiltonian must satisfy the constraint

$$
\Delta t D_{0}\left(H_{1}, H_{2}\right) \geq \pi .
$$

where $D_{0}\left(H_{1}, H_{2}\right)$ measures how much the two Hamiltonians differ and is defined as follows. Take the difference of the two Hamiltonians $H^{d}=H_{1}-H_{2}$. Denote by $E_{\max }^{d}$ and $E_{\min }^{d}$ the largest and smallest eigenvalue of $H^{d}$ respectively. Then $D_{0}\left(H_{1}, H_{2}\right)$ is given by

$$
D_{0}\left(H_{1}, H_{2}\right)=\max \left\{E_{\text {max }}^{d}-E_{\text {min }}^{d},\left|E_{\text {max }}^{d}\right|,\left|E_{\text {min }}^{d}\right|\right\} .
$$

\section{(This expression generalises a result of [5]).}

In the appendix it is shown that $D_{0}$ is a distance on the space of Hamiltonian operators (ie. it is symmetric, positive and equal to zero only if the two Hamiltonians coincide, and it obeys the triangle inequality). This important property is central to some of the arguments below.

In order to make the problem considered by Childs, Preskill and Renes more concrete, consider the following example. You are given a box in which there is one of two unknown magnetic fields $\vec{B}_{1}$ or $\vec{B}_{2}$. Your task is to determine which kind of box you have. The only way you can probe the box is to send through the box a specific kind of particle of magnetic moment $\mu$. Thus the two boxes differ in that they act as two different Hamiltonians $H_{1,2}=\mu \vec{B}_{1,2} \cdot \vec{\sigma}$. Given sufficient time or a sufficient supply of particles you can always determine what is the magnetic field. (We suppose that the time that the particles pass in the box can be freely chosen, for instance by choosing their initial velocity. And one can send the particle through the box as many times as one wants). However if you are given only one particle, what is the minimum time necessary to accomplish this task? Childs, Preskill and Renes show that this time is limited by eq. (2).

We now present a proof of eqs. (2, 3) that is inspired by the techniques developed to study interaction free measurements in [6]. The proof is also closely connected to the bounds on oracle query complexity obtained in [7] and even more so to a continuous-time analogue obtained in [8]. We first describe the most general strategy that could be used. First of all, we consider that we may let the particle go into the box or not, i.e. for certain values of its position the particle can pass through the box and for others not. This allows us to make a superposition of particle passing through the box and not passing through the box. Mathematically we describe this by the decomposition of the Hilbert space into the sum $\mathcal{H}=\mathcal{H}_{\text {box }} \oplus \mathcal{H}_{\text {Nbox }}$ where $\mathcal{H}_{\text {box }}$ corresponds to the particle passing through the box and $\mathcal{H}_{N b o x}$ corresponds to the particle passing next to the box. Second, we consider that the particle can also be entangled with some other particle, called ancilla, onto which the hamiltonian doesn't act (eg. the ancilla is kept out of the box). The Hilbert space is thus further increased to $\mathcal{H}=\left(\mathcal{H}_{\text {box }} \oplus \mathcal{H}_{\text {Nbox }}\right) \otimes \mathcal{H}_{\text {ancilla }}$.

In the particular problem considered in this section, namely, distinguishing between two possible Hamiltonians, using an ancilla turns out to be irrelevant. (However when one must distinguish between more than two Hamiltonians, using an ancilla can be helpfull, see [5] for a simple example). To simplify the proof we will first consider the case with no ancilla and then show in section II that the presence of the ancilla makes no difference.

The most general strategy consists of sending the particle several times through the box and making an arbitrary unitary transformation on the particle after each passage. We describe this as follows. Initially the particle is in state $\left|\psi_{0}\right\rangle$. Before the $\mathrm{k}$-th passage through the box the particle is in state $\left|\psi_{k-1}^{i}\right\rangle$ where the superscript $i=1,2$ corresponds to which Hamiltonian $H_{1,2}$ is acting on the particle. We decompose the state as $\left|\psi_{k-1}^{i}\right\rangle=\left|u_{k}^{i}\right\rangle+\left|v_{k}^{i}\right\rangle$ where $\left|u_{k}^{i}\right\rangle \in \mathcal{H}_{\text {box }}$ and $\left|v_{k}^{i}\right\rangle \in \mathcal{H}_{\text {Nbox }}$. After passing through the box the particles state is $\left|\psi_{k}^{\prime i}\right\rangle=e^{-i H_{i} t_{k}}\left|u_{k}^{i}\right\rangle+\left|v_{k}^{i}\right\rangle$ where $t_{k}$ is the time the particle is in the box (and we have supposed without loss of generality that if the particle does not pass through the box the Hamiltonian is zero). We then make an arbitrary unitary transformation on the particle $\left|\psi_{k}^{\prime i}\right\rangle \rightarrow\left|\psi_{k}^{i}\right\rangle=U_{k}\left|\psi_{k}^{\prime i}\right\rangle$. We can describe the whole evolution succinctly by

$$
\left|\psi_{k}^{i}\right\rangle=U_{k} e^{-i \tilde{H}_{i} t_{k}}\left|\psi_{k-1}^{i}\right\rangle
$$


where $\tilde{H}_{i}$ is the extension of $H_{i}$ to $\mathcal{H}_{\text {box }} \oplus \mathcal{H}_{N b o x}$. It is equal to $H_{i}$ on $\mathcal{H}_{\text {box }}$ and equal to zero on $\mathcal{H}_{\text {Nbox }}$.

We therefore have

$$
\left\langle\psi_{k}^{1} \mid \psi_{k}^{2}\right\rangle=\left\langle\psi_{k-1}^{1}\left|e^{i \tilde{H}_{1} t_{k}} e^{-i \tilde{H}_{2} t_{k}}\right| \psi_{k-1}^{2}\right\rangle,
$$

or expressed differently

$$
\left\langle\psi_{k}^{1} \mid \psi_{k}^{2}\right\rangle-\left\langle\psi_{k-1}^{1} \mid \psi_{k-1}^{2}\right\rangle=\left\langle\psi_{k-1}^{1}\left|e^{i \tilde{H}_{1} t_{k}} e^{-i \tilde{H}_{2} t_{k}}-1\right| \psi_{k-1}^{2}\right\rangle .
$$

The procedure to distinguish between $H_{1}$ and $H_{2}$ can only improve if the total time $T$ is kept fixed, but the particle is allowed to pass more times through the box. That is the discrimination can only improve if one takes smaller time intervals $t_{k}$. In the limit of infinitesimal $t_{k}$ one obtains

$$
\frac{d\left\langle\psi^{1} \mid \psi^{2}\right\rangle}{d t}=i\left\langle\psi^{1}\left|\left(\tilde{H}_{1}-\tilde{H}_{2}\right)\right| \psi^{2}\right\rangle=i\left\langle\psi^{1}\left|\tilde{H}^{d}\right| \psi^{2}\right\rangle
$$

where $\tilde{H}^{d}=\tilde{H}_{1}-\tilde{H}_{2}$ is equal to $H^{d}=H_{1}-H_{2}$ on $\mathcal{H}_{b o x}$ and equal to zero on $\mathcal{H}_{\text {Nbox }}$. The absolute value of the overlap therefore changes as

$$
\frac{d\left|\left\langle\psi^{1} \mid \psi^{2}\right\rangle\right|^{2}}{d t}=4 \operatorname{Im}\left[\left\langle\psi^{2} \mid \psi^{1}\right\rangle\left\langle\psi^{1}\left|\tilde{H}^{d}\right| \psi^{2}\right\rangle\right] .
$$

We can always write the states $\left|\psi^{1,2}\right\rangle$ as

$$
\begin{gathered}
\left|\psi^{1}\right\rangle=e^{+i \chi / 2}\left(\cos (\theta / 2)\left|\psi^{\|}\right\rangle+\sin (\theta / 2)\left|\psi^{\perp}\right\rangle\right) \\
\left|\psi^{2}\right\rangle=e^{-i \chi / 2}\left(\cos (\theta / 2)\left|\psi^{\|}\right\rangle-\sin (\theta / 2)\left|\psi^{\perp}\right\rangle\right) \\
0 \leq \theta \leq \pi / 2 \quad, \quad\left\langle\psi^{\|} \mid \psi^{\perp}\right\rangle=0
\end{gathered}
$$

This enables us to write (8) as

$$
\frac{d \cos ^{2} \theta}{d t}=2 \cos \theta \sin \theta \operatorname{Im}\left[\left\langle\psi^{\|}\left|\tilde{H}^{d}\right| \psi^{\perp}\right\rangle\right]
$$

or equivalently

$$
\frac{d \theta}{d t}=\operatorname{Im}\left[\left\langle\psi^{\|}\left|\tilde{H}^{d}\right| \psi^{\perp}\right\rangle\right]
$$

Now we note that for any operator $A$,

$$
\left|\operatorname{Im}\left[\left\langle\psi^{\|}|A| \psi^{\perp}\right\rangle\right]\right| \leq \frac{a_{\max }-a_{\min }}{2}
$$

where $a_{\max }$ is the largest eigenvalue of $A$ and $a_{\min }$ the smallest. Equality is attained if

$$
\begin{aligned}
\left|\psi^{\|}\right\rangle & =\frac{e^{i \varphi}\left|a_{\max }\right\rangle+e^{i \varphi^{\prime}}\left|a_{\min }\right\rangle}{\sqrt{2}} \\
\left|\psi^{\perp}\right\rangle & =\frac{e^{i(\varphi+\pi / 2)}\left|a_{\max }\right\rangle+e^{i\left(\varphi^{\prime}-\pi / 2\right)}\left|a_{\min }\right\rangle}{\sqrt{2}}
\end{aligned}
$$

where $\varphi$ and $\varphi^{\prime}$ are arbitrary phases. In the case of $\tilde{H}^{d}$, its largest eigenvalue is $\max \left\{E_{\text {max }}^{d}, 0\right\}$ and its smallest eigenvalue is $\min \left\{E_{\min }^{d}, 0\right\}$ (where $E_{\max }^{d}$ and $E_{\text {min }}^{d}$ are the largest and smallest eigenvalues of $H^{d}$ ). Hence the absolute value of the right hand side of (11) is bounded by $\left|\operatorname{Im}\left[\left\langle\psi^{\|}\left|\tilde{H}^{d}\right| \psi^{\perp}\right\rangle\right]\right| \leq D_{0}\left(H_{1}, H_{2}\right) / 2$ where $D_{0}$ is given by (3). We therefore have

$$
\left|\frac{d \theta}{d t}\right| \leq D_{0}\left(H_{1}, H_{2}\right) / 2
$$

Integrating we have

$$
-\frac{D_{0}\left(H_{1}, H_{2}\right) t}{2} \leq \theta(t)-\theta(0) \leq \frac{D_{0}\left(H_{1}, H_{2}\right) t}{2} .
$$

Initially $\theta(0)=0$ since $|\psi(0)\rangle$ is independent of $i$. Requiring that one can recognize with certainty which is the Hamiltonian, that is requiring $\theta(t)=\pi / 2$, one finds that $t D_{0}\left(H_{1}, H_{2}\right) \geq \pi$, as announced.

In the above proof the possibility of letting the particle go through the box as well as outside the box allows us to extend the Hamiltonian $H^{d}$ so that it also has the eigenvalue zero. This is described by replacing $H_{i}$ with $\tilde{H}_{i}$. It is the possibility that allows the maximum in eq. (3) to be taken not only over the first term $E_{\max }^{d}-$ $E_{\text {min }}^{d}$, but also over the two other terms, $\left|E_{\text {max }}^{d}\right|$ and $\left|E_{\text {min }}^{d}\right|$ and makes our result eq. (3) differ from the result obtained in [5]. Indeed if one does not allow for this then eq. (2) continues to hold, but eq. (3) is replaced by $D_{0}\left(H_{1}, H_{2}\right)=E_{\max }^{d}-E_{\min }^{d}$. Thus in this case the measurement may be less efficient.

In order to illustrate how the "in/out of the box" possibility can be used, we consider the following example. We are given one of two black boxes, and must distinguish which box we have. The boxes are conducting and hollow. They are connected to an external electrostatic potential. The only way the two boxes differ is that the potential can take two different values $\phi_{1}$ and $\phi_{2}$. The only way we can probe which box we have is by sending a charged particle through the box. The particle will therefore acquire a phase which depends on the potential. Mathematically we can describe this as the problem of distinguishing two Hamiltonians $H_{1,2}=H_{0}+\phi_{1,2} I$ where $I$ is the identity operator. In order to distinguish which box we have, we put the particle in a superposition of two states, one of which passes through the box and the other which does not. Thus we prepare the particle in the state $(\mid$ through box $\rangle+\mid$ Not through box $\rangle) / \sqrt{2}$ and send the particle at a speed such that it passes a time $T=\pi /\left(\phi_{1}-\phi_{2}\right)$ in the box. After this time the particle is in one of two orthogonal states (| Not through box $\rangle \pm$ $e^{i \pi \phi_{1} /\left(\phi_{1}-\phi 2\right)} \mid$ through box $\left.\rangle\right) / \sqrt{2}$ which can easily be distinguished. On the other hand, if we had not been allowed to use the "out of the box" alternative, we could not have distinguished between the 2 Hamiltonians since they differ only by a constant term which adds an unobservable phase to the wave function.

There are also situations where it is not necessary to use the "in/out of the box" possibility. Suppose one must distinguish, using a spin $1 / 2$ particle of magnetic moment $\mu$, between two magnetic fields of equal magnitude but pointing in opposite directions $B_{1,2}= \pm B_{0} \overrightarrow{1}_{z}$. The strategy in this case is simply to prepare the spin in state $\left|\uparrow_{x}\right\rangle$ 
and let it evolve in the magnetic field a time $T=\pi / \mu B_{0}$ and then to measure the operator $\sigma_{y}$.

\section{B. Distinguishing between two time dependent Hamiltonians}

In the preceeding section we considered the case where the two Hamiltonians which must be distinguished are time independent. One can easily generalize this result to the case where the Hamiltonians are time dependent.

Let us suppose one must distinguish between two time dependent Hamiltonians $H_{1}(t)$ and $H_{2}(t)$. The arguments of section IIA can be followed unchanged until eq. (14) which becomes

$$
\left|\frac{d \theta}{d t}\right| \leq D_{0}\left(H_{1}(t), H_{2}(t)\right) / 2
$$

where $D_{0}\left(H_{1}(t), H_{2}(t)\right)$ is the instantaneous value of the distance eq. (3). Integrating we have

$$
\begin{aligned}
-\int_{0}^{t} d t \frac{D_{0}\left(H_{1}(t), H_{2}(t)\right)}{2} & \leq \theta(t)-\theta(0) \\
& \leq \int_{0}^{t} d t \frac{D_{0}\left(H_{1}(t), H_{2}(t)\right)}{2} .
\end{aligned}
$$

Initially $\theta(0)=0$ since $|\psi(0)\rangle$ is independent of $i$. Requiring that one can recognize with certainty which is the Hamiltonian, that is requiring $\theta(t)=\pi / 2$, one finds that

$$
\int_{0}^{t} d t D_{0}\left(H_{1}(t), H_{2}(t)\right) \geq \pi
$$

which is the generalization of the constraint eq. (2) to the case of time dependent Hamiltonians.

\section{Using an ancilla does not help to distinguish between two Hamiltonians}

In the proof of eqs. (2, 3) and (18) given in sections II A and II B we did not consider the possibility that the particle passing through the box is entangled with another particle (ancilla) was not considered. We shall now show that if we assume the ancilla is not allowed into the box, then eqs. (2, 3) and (18) continue to hold.

To this end it is helpfull to view the particle and ancilla as a single larger system with Hamiltonian $H_{i}^{\text {total }}=$ $H_{i}(t) \otimes H_{\text {ancilla }}(t)$ where $H_{i}$ is unknown and $H_{\text {ancilla }}$ is known but arbitrary, and to reformulate the task as the problem of distinguishing $H_{1}^{\text {total }}$ from $H_{2}^{\text {total }}$. Note that this reformulation englobes apparently more involved strategies where for instance the ancilla is repeatedly measured and the evolution made conditional on the results of these intermediate measurements. Indeed by including the measuring device in an even bigger ancilla one recovers the above formulation.
Now the distance $D_{0}$ between two such Hamiltonians obeys

$$
\begin{gathered}
D_{0}\left(H_{1}^{\text {total }}(t), H_{2}^{\text {total }}(t)\right) \\
=D_{0}\left(H_{1}(t) \otimes H_{\text {ancilla }}(t), H_{2}(t) \otimes H_{\text {ancilla }}(t)\right) \\
=D_{0}\left(H_{1}(t), H_{2}(t)\right) .
\end{gathered}
$$

Thus the time required to distinguish between the two Hamiltonians eq. (18) does not depend on the presence of the ancilla.

The basic reason why an ancilla does not help in distinguishing between two unknown Hamiltonians is that all the argument of section II A depends on the eigenvalues of the difference between the two Hamiltonians $H^{d}$ but not on the degeneracy of the eigenvalues. Including and ancilla does not change the eigenvalues of $H^{d}$, but changes their degeneracy.

\section{Attaining the bound in the dichotomic case}

We shall now show that one can always attain the bound eq. (2). In general this will require putting the particle in a superposition of "in the box" and "out of the box" states. Equation (13) shows that to attain the bound the particle must be kept in a superposition with equal weights of the eigenstates of $\tilde{H}^{d}=\tilde{H}_{1}^{d}-\tilde{H}_{2}^{d}$ with maximal and minimal eigenvalue, $\left|\tilde{E}_{\text {max }}^{d}\right\rangle$ and $\left|\tilde{E}_{\text {min }}^{d}\right\rangle$. In the preceeding section we gave two examples of how to do this when the two Hamiltonians $H_{1}$ and $H_{2}$ commute. When the Hamiltonians do not commute one must use a more complicated strategy. Let us first rewrite the Hamiltonians $\tilde{H}_{1}$ and $\tilde{H}_{2}$ as

$$
\begin{array}{ccc}
\tilde{H}_{1}=\tilde{H}^{+}+\tilde{H}^{d} / 2 & , & \tilde{H}_{2}=\tilde{H}^{+}-\tilde{H}^{d} / 2 \\
\tilde{H}^{+}=\left(\tilde{H}_{1}+\tilde{H}_{2}\right) / 2 & , & \tilde{H}^{d}=\tilde{H}_{1}-\tilde{H}_{2} .
\end{array}
$$

The evolution during a small time interval $\tau$ can then be written as

$$
\begin{aligned}
\exp \left(-i \tilde{H}_{1} \tau\right) & =\exp \left(-i\left(\tilde{H}^{+}+\tilde{H}^{d} / 2\right) \tau\right) \\
& \simeq \exp \left(-i \tilde{H}^{+} \tau\right) \exp \left(-i \tilde{H}^{d} \tau / 2\right) \exp \left(O\left(\tau^{2}\right)\right) \\
\exp \left(-i \tilde{H}_{2} \tau\right) & =\exp \left(-i\left(\tilde{H}^{+}-\tilde{H}^{d} / 2\right) \tau\right) \\
& \simeq \exp \left(-i \tilde{H}^{+} \tau\right) \exp \left(+i \tilde{H}^{d} \tau / 2\right) \exp \left(O\left(\tau^{2}\right)\right) .
\end{aligned}
$$

To distinguish the two Hamiltonians we initially prepare the system in the state $|\psi(0)\rangle=\left(\left|\tilde{E}_{\text {max }}^{d}\right\rangle+\left|\tilde{E}_{\text {min }}^{d}\right\rangle\right) / \sqrt{2}$. The evolution is the following. We let the unknown Hamiltonian act for a small time $\tau=T / N$ where $T=$ $\pi / D_{0}\left(H_{1}, H_{2}\right)$ and $N$ is a large integer. We then act on the system with the unitary transformation $U=$ $\exp \left(-i \nu \tilde{H}^{d}\right) \exp \left(+i \tilde{H}^{+} \tau\right)$ where $\nu$ is an arbitrary real number. The term on the right in $U$ cancels the term on the left in eq. (21). This unitary evolution $U$ can in principle be done in an arbitrarily short time. After $N$ 
repetitions, the evolution is

$$
\begin{aligned}
|\psi(T)\rangle & =\left[\exp \left(-i(\nu \pm 1 / 2) \tilde{H}^{d} \tau\right) \exp \left(O\left(\tau^{2}\right)\right)\right]^{N}|\psi(0)\rangle \\
& \simeq \exp \left(-i \pi(\nu \pm 1 / 2) \tilde{H}^{d}\right)|\psi(0)\rangle
\end{aligned}
$$

where we have written an expression valid in the limit of large $N$ Thus we obtain two orthogonal states which can be distinguished with certainty.

Note that if we take the arbitrary real number $\nu$ to be either $\pm 1 / 2$, then $U=\exp \left(-i \tau \tilde{H}_{1,2}\right)$, that is we have exactly canceled the evolution of one of the Hamiltonians. This is the technique that is proposed in [5].

\section{ESTIMATING AN UNKNOWN HAMILTONIAN}

\section{A. Formulation of the problem}

In the previous section we considered the situation where one must distinguish with certainty between two possible Hamiltonians. In the present section we shall consider the problem where one must estimate with finite precision what is the Hamiltonian.

In order to give a precise formulation to this problem, let us suppose that the possible Hamiltonians are denoted $H_{i}$ and can occur with a priori probabilities $p\left(H_{i}\right)$. After an evolution that lasts for a time $\Delta t$, a measurement is carried out which yields result $j$. The probability of result $j$ given that the Hamiltonian is $H_{i}$ is denoted $p\left(j \mid H_{i}\right)$. The result of the measurement allows one to estimate what is the Hamiltonian. We shall suppose that one makes a guess of what is the true Hamiltonian. Call the guess $H_{j}^{\text {guess }}$. Note that $H_{j}^{\text {guess }}$ can be one of the initial Hamiltonians $H_{i}$, or it could be a different Hamiltonian altogether. The quality of the guess is measured by an uncertainty

$$
\Delta H=\sum_{i} p\left(H_{i}\right) \sum_{j} p\left(j \mid H_{i}\right) D\left(H_{i}, H_{j}^{\text {guess }}\right) .
$$

In this equation $D\left(H_{i}, H_{j}^{\text {guess }}\right)$ is a distance on the space of Hamiltonians that measures how close the guess is to the true Hamiltonian $H_{i}$. It is natural to normalize the distance $D$, for instance by requiring that $D(H, H+E \mathbb{1})=E$.

There are many different distances on the space of Hamiltonians. For instance $D\left(H_{1}, H_{2}\right)=$ $\sqrt{\operatorname{Tr}\left(H_{1}-H_{2}\right)^{2}} / d$ where $d$ is the dimension of the Hilbert space. In general the distance that one will use will depend on the specific problem one considers. In the present paper we shall use the distance $D_{0}$ introduced in eq. (3). The reason is that this is the distance that appears in the results of section II, and these results are used in the arguments below. We expect that using another distance would change quantitatively, but not qualitatively, our results.
One of the most fundamental questions concerning the estimation of an unknown Hamiltonian is the relation between the precision $\Delta H$ with which the Hamiltonian is known and the time $\Delta t$ used to carry out the estimation. The remainder of this section is devoted to addressing this question.

\section{B. Estimating a Hamiltonian which can only take two values, $H_{1}$ or $H_{2}$}

As a first application of the general problem of estimating an unknown Hamiltonian consider the particular situation in which their are only two possible Hamiltonians, $H_{1}$ and $H_{2}$, which are equally probably $\left(p\left(H_{1}\right)=\right.$ $\left.p\left(H_{2}\right)=1 / 2\right)$ and the task is to estimate which it is in a finite time $\Delta t$. The quality of this estimate shall be expressed by using as distance on the space of Hamiltonians the distance $D_{0}$ defined in eq. (3). The techniques developed in section [I] will allow us to solve this problem exactly.

In section II it was shown that if $\Delta t \geq \pi / D_{0}\left(H_{1}, H_{2}\right)$, then the two Hamiltonians can be distinguished perfectly and therefore $\Delta H$ is zero. On the other hand when $\Delta t \rightarrow 0$, it is impossible to obtain any information about the Hamiltonians and the best strategy is to randomly guess either $H_{1}$ or $H_{2}$. Hence in this limit $\Delta H \rightarrow D_{0}\left(H_{1}, H_{2}\right) / 2$. For intermediate times $\Delta H$ will decrease from $D_{0}\left(H_{1}, H_{2}\right) / 2$ to zero as a function of $\Delta t$. We shall show that for the optimal estimation strategy, $\Delta H$ is given by

$$
\Delta H=\max \left\{0, \frac{D_{0}\left(H_{1}, H_{2}\right)}{2}\left(1-\sin \left(\frac{D_{0}\left(H_{1}, H_{2}\right) \Delta t}{2}\right)\right)\right\} .
$$

To prove this let us first show that for an optimal guessing strategy it is sufficient that the guessed Hamiltonian be either $H_{1}$ or $H_{2}$. We recall that in the estimation problem as formulated in section IIIA we left open the possibility of guessing a Hamiltonian that is not one of the possible Hamiltonians $H_{i}$. In the present case it is not necessary to consider such possibilities.

To show this let us consider the contribution of guess $j$ to $\Delta H$ (we denote this contribution $\Delta H(j)$ ):

$$
\begin{aligned}
\Delta H(j)= & \frac{1}{2} p\left(j \mid H_{1}\right) D\left(H_{1}, H_{j}^{\text {guess }}\right) \\
& +\frac{1}{2} p\left(j \mid H_{2}\right) D\left(H_{2}, H_{j}^{\text {guess }}\right) .
\end{aligned}
$$

Using the triangle inequality we can write

$$
\begin{aligned}
\Delta H(j) \geq & \frac{1}{2} p\left(j \mid H_{1}\right) D\left(H_{1}, H_{2}\right) \\
& +\frac{1}{2}\left[p\left(j \mid H_{2}\right)-p\left(j \mid H_{1}\right)\right] D\left(H_{2}, H_{j}^{\text {guess }}\right)
\end{aligned}
$$

If $p\left(j \mid H_{2}\right)-p\left(j \mid H_{1}\right) \geq 0$ we finally have

$$
\Delta H(j) \geq \frac{1}{2} p\left(j \mid H_{1}\right) D\left(H_{1}, H_{2}\right)
$$


with equality if and only if $H_{j}^{\text {guess }}=H_{2}$. Thus if the a posteriori probability that the Hamiltonian was $H_{2}$ is greater than the a posteriori probability that the Hamiltonian was $H_{1}$ one should guess that the Hamiltonian is $H_{2}$. And conversely if the a posteriori probability that the Hamiltonian was $H_{1}$ is greater than the a posteriori probability that the Hamiltonian was $\mathrm{H}_{2}$ one should guess that the Hamiltonian is $H_{1}$.

Let us now consider the optimal evolution and measurement strategy. The estimation strategy starts with a given quantum state $\psi_{0}$. If the Hamiltonian is $H_{1}$ this state evolves into $\psi_{1}(t)$ whereas if the Hamiltonian is $H_{2}$ the state evolves into $\psi_{2}(t)$. In section $\amalg \mathrm{IA}$ it was shown that the overlap between these two states must obey the inequality

$$
\left|\left\langle\psi_{1}(t) \mid \psi_{2}(t)\right\rangle\right| \geq \cos \frac{D_{0} t}{2}
$$

with equality attained for the optimal strategy (we have denoted $D_{0}=D_{0}\left(H_{1}, H_{2}\right)$ ). Furthermore it is shown in [10] that the probability $p_{E}$ of making an error when trying to distinguish two equiprobable states $\psi_{1}$ and $\psi_{2}$ is bounded by

$$
p_{E} \geq \frac{1-\sqrt{1-\left|\left\langle\psi_{1} \mid \psi_{2}\right\rangle\right|^{2}}}{2} \geq \frac{1-\sin \left(D_{0} t / 2\right)}{2}
$$

with equality attained if one carries out a von Neumann measurement of the basis $\left(\left|\psi^{\|}\right\rangle \pm\left|\psi^{\perp}\right\rangle\right) / 2$. Hence we find that for the optimal strategy

$$
\Delta H=p_{E} D_{0}=\frac{D_{0}}{2}\left(1-\sin \left(\frac{D_{0} \Delta t}{2}\right)\right)
$$

which proves eq. (24).

\section{Estimating a completely unknown Hamiltonian}

We now consider the situation where the Hamiltonian is completely unknown. Once more we shall use as distance on the space of Hamiltonians the distance $D_{0}$ of eq. (3). We shall show that in this case the precision $\Delta H$ with which the Hamiltonian is estimated and the time used to estimate the Hamiltonian must obey the constraint

$$
\Delta H \Delta t \geq \frac{1}{4} .
$$

This constitutes one of the fundamental results of this paper.

To prove this, we will contrast two situations. In the first situation (which is the one we are interested in) the experimenter has no information about the Hamiltonian. In the second situation we imagine that there is a "spy" that knows the true Hamiltonian, call it $H_{0}$. The spy then tells the experimenter that the true Hamiltonian is either $H_{0}$ or some other Hamiltonian $H_{1}$. The a priori probabilities that the spy chooses Hamiltonian $H_{0}$ or $H_{1}$ are equal.

Denote by $\Delta H$ the maximum precision with which the Hamiltonian can be known in the first case and by $\Delta H_{\text {dicho }}$ the maximum precision with which the Hamiltonian can be known with the help of the spy. Obviously $\Delta t \Delta H \geq \Delta t \Delta H_{\text {dicho }}$ since the time intervals are the same in the two situations and the information provided by the spy can only increase the precision with which one can estimate the Hamiltonian. This means that $\Delta t \Delta H \geq \max \Delta t \Delta H_{\text {dicho }}$ where the maximum is taken over all possible choices of the spy. The results of section IIIB can be used to show that $\max \Delta t \Delta H_{\text {dicho }} \geq 1 / 4$, which proves eq. (31).

To show that max $\Delta t \Delta H_{\text {dicho }} \geq 1 / 4$, note that in the dichotomic case, the product $\Delta H \Delta t$ takes the form

$$
\Delta H \Delta t=\frac{D_{0} \Delta t}{2}\left(1-\sin \left(\frac{D_{0} \Delta t}{2}\right)\right)
$$

for $0 \leq \Delta t \leq \pi / D_{0}$. For small times this tends to zero since $\Delta H$ is bounded and $\Delta t \rightarrow 0$. And for $\Delta t \geq \pi / D_{0}$ the product is zero since $\Delta H=0$. There is an intermediate time where the product attains its maximum. One easily shows (using $\sin x \leq x$ for $x \geq 0$, which implies that $x(1-\sin x) \geq x(1-x))$ that the maximum value of this product is greater than a quarter:

$$
\max \Delta H \Delta t \geq \frac{1}{4}
$$

\section{Estimating a completely unknown Hamiltonian acting in a $d$ dimensional space}

Equation (31) gives a lower bound on the product of the precision with which a completely unkown Hamiltonian is measured and the time taken to estimate it. We beleive that this lower bound is not tight and that in general a stronger lower bound should hold. We do not know at present what form this stronger lower bound will take, but we beleive that it should depend on the dimensionality of the Hilbert space on which the Hamiltonian acts.

An indication that this should be the case is provided by an example due to Farhi and Gutmann [8] inspired by Grover's search algorithm 9 . In this example one must distinguish between $d$ Hamiltonians of the form $H_{k}=$ $E|k\rangle\langle k|$ where the $d$ states $|k\rangle$ form an orthonormal basis. Farhi and Gutmann show that in order to distinguish these Hamiltonians perfectly, a minimum time of $\Delta t \geq$ $c d^{1 / 2} / E$ is necessary (where $c$ is some positive constant).

This example shows that there are situations where estimating an unkown Hamiltonian becomes increasingly difficult as the dimension $d$ of the Hilbert space on which it acts increases. However in the Fahri-Gutmann example, the unkown Hamiltonian has a very specific form which is known before hand. We have obtained preliminary indications that when the Hamiltonian is completely unkown, estimating it should take substantially 
more time than sugested by the Fahri-Gutmann example. We hope to report on this issue in a future publication.

\section{MEASURING ENERGY WHEN THE HAMILTONIAN IS UNKNOWN}

\section{A. Introduction}

The results presented in the preceding section concerning the estimation of Hamiltonians have important implications for energy measurements. As shown in [3] the energy of the state can be measured in an arbitrarily short time. However a careful scrutiny of the arguments of [3] shows that a quick energy measurement is possible only if the Hamiltonian is known. In the example discussed in the introduction, it is possible to carry out a quick energy measurement only because we know that the particle is in a magnetic field $\vec{B}_{0}$ of known magnitude pointing along the $+z$ direction. Suppose however that the magnetic field is pointing initially either along the $+z$ or the $-z$ axis. Then a measurement of $\sigma_{z}$ yields no information about the energy. Thus in order to determine the energy of the particle, we must also determine the magnetic field. That is we must also determine what is the Hamiltonian. But as we discussed above, determining the Hamiltonian will take a minimum time $\Delta t$. (We suppose that the only way we can probe the magnetic field is with a particle of magnetic moment $\mu$. Of course if we could use a particle of larger magnetic moment, the measurement of the magnetic field could be done faster). Thus in this example measuring the energy of the state cannot be done instantaneously because the Hamiltonian is not perfectly known.

In fact this is a very general result. We shall show below that if the Hamiltonian acting on a system is completely unknown, then the precision $\Delta E$ with which one can estimate the energy of the state in a time interval $\Delta t$ obeys the constraint

$$
\Delta t \Delta E \geq 1 / 4
$$

This assertion follows easily from the results obtained in the previous section. However before proving it we first need to define with precision what we mean by accuracy $\Delta E$ of an energy measurement.

\section{B. Accuracy of an energy measurement}

An ideal energy measurement is a von Neumann measurement of the Hamiltonian operator $H=\sum_{E} E|E\rangle\langle E|$. If the quantum state is $|\psi\rangle$, the measurement gives result $E$ with probability $p(E \mid \psi)=|\langle\psi \mid E\rangle|^{2}$.

Let us consider an imperfect measurement. This measurement will predict that the energy is $E^{\prime}$ with probability $p\left(E^{\prime}\right)$. Neither the energies $E^{\prime}$ nor the probabilities $p\left(E^{\prime}\right)$ need coincide with the energies and probabilities for an ideal energy measurement. Nevertheless we would like to define in a precise way the accuracy of an energy measurement.

The simplest situation in which to define the accuracy of an energy measurement is when the quantum state is an energy eigenstate $|\psi\rangle=|E\rangle$. In this case the true energy of the state is well defined. Hence the accuracy of the imperfect energy measurement is simply the amount by which the energies $E^{\prime}$ differ from the true energy $E$ :

$$
\Delta E=\sum_{E^{\prime}} p\left(E^{\prime} \mid E\right)\left|E^{\prime}-E\right|
$$

where $p\left(E^{\prime} \mid E\right)$ is the probability that the estimated energy is $E^{\prime}$ when the quantum state is $\psi=|E\rangle$.

If the state is not an energy eigenstate then we define the accuracy of the energy measurement as the average over the probability $|\langle E \mid \psi\rangle|^{2}$ that an ideal energy measurement gives result $E$ times of the accuracy of the measurement if the state is $|E\rangle$ :

$$
\Delta E=\sum_{E}|\langle E \mid \psi\rangle|^{2} \sum_{E^{\prime}} p\left(E^{\prime} \mid E\right)\left|E^{\prime}-E\right| .
$$

\section{Proof of the time-energy uncertainty relation for energy measurements when the Hamiltonian is completely unknown}

We start by noting that the proof of eq. (34) when $\psi$ is not an eigenstate of the Hamiltonian follows from the case where $\psi$ is an eigenstate of the Hamiltonian since we have defined in (36) the uncertainty when $\psi$ is not an eigenstate of the Hamiltonian as the average of the uncertainties when $\psi$ is an eigenstate of the Hamiltonian times the probability that a measurement of the Hamiltonian operator yields the corresponding energy. Therefore we can restrict ourselves to considering the case where $\psi=|E\rangle$ is an eigenstate of the Hamiltonian $H$. Since the Hamiltonian $H$ is completely unknown, the state $\psi$ is also unknown.

To prove eq. (34) we fix $\Delta t$ and contrast as in the section III , two situations. In the first one has no information about the Hamiltonian. In the second a spy gives the additional information that the Hamiltonian is either $H_{0}$ (the true Hamiltonian) or $H_{1}$. We shall suppose that $H_{1}=H_{0}+\epsilon \mathbb{1}$ where $\epsilon$ is a c-number and $\mathbb{1}$ is the identity operator. We shall further suppose that with the information provided by the spy there is equal a priori probabilities that the Hamiltonian is $H_{0}$ or $H_{1}$. Let us denote the energy uncertainty in the first situation by $\Delta E$ and in the second situation by $\Delta E_{\text {spy }}$. Obviously we have $\Delta t \Delta E \geq \Delta t \Delta E_{s p y}$ since $\Delta t$ is the same in both situations and the information provided by the spy can only decrease the energy uncertainty. We therefore want to put a bound on $\Delta E_{\text {spy }}$.

First note that since the two Hamiltonians $H_{0}$ and $H_{1}=H_{0}+\epsilon \mathbb{1}$ commute the experimenter can immediately determine what is the state $\psi$ by measuring the 
operator $H_{0}$. This measurement can in principle be done arbitrarily fast. Hence the experimenter knows that the energy is either $E$, the true energy, or $E+\epsilon$.

Since the experimenter has only two possibilities between which to choose, an optimal strategy will consist of guessing either that the energy is $E$ or $E+\epsilon$. It is not necessary to consider other possibilities such as guessing that the energy is $E+\epsilon / 2$. Furthermore the energy uncertainty will be $\Delta E_{s p y}=\epsilon p_{E}$ where $p_{E}$ is the probability of making the wrong guess. The proof of these assertions follows from the fact that $\Delta E$, as defined in eq. (35), is linear in the probabilities $p\left(E^{\prime}\right)$ times a distance $\left|E^{\prime}-E\right|$ on the space of energies. Hence the arguments of section III B, eqs 25 to 27, can be used in the present case.

But the error probability $p_{E}$ of mistaking one energy for the other is identical to the error probability of wrongly identifying the Hamiltonians $H_{0}$ and $H_{1}$ (since knowing the energy is equivalent to knowing the Hamiltonian). Hence in the present case $\Delta E_{s p y}=\Delta H$ where $\Delta H$ is the uncertainty in estimating the two Hamiltonians $H_{0}$ and $H_{1}$. But we have shown in section IIIB that for given $\Delta t$ there exists a choice of $\epsilon$ such that $\Delta t \Delta H \geq 1 / 4$.

\section{Saturating the time-energy uncertainty for energy measurements when the Hamiltonian is unknown?}

We now address the question of whether it is possible to devise a universal measurement strategy that can determine the energy of an unknown state even if there is no prior knowledge about the Hamiltonian? Such universal measurement strategies exist and are well known. We illustrate them by a typical example, namely the emission of electromagnetic radiation by an excited state of an atom.

We then inquire whether this measurement strategy saturates the time-energy uncertainty relation obtained in the previous section. It turns out that for this measurement strategy, and using the definition of section IV B, $\Delta t \Delta E$ is infinite. However in a qualitative way this measurement strategy does obey a time energy uncertainty. This is discussed in detail.

As mentioned in the introduction the emission of one or more photons by an excited atomic state can be viewed as a measurement of the energy of the electrons. The coupling between the measuring apparatus (the electromagnetic field $A^{\mu}$ ) and the system (the electron) is realized through the interaction

$$
H_{\text {int }}=\int d^{3} x A^{\mu}(x) J_{\mu}(x)
$$

where $J_{\mu}(x)$ is the electric current. In interaction representation it takes the form

$$
H_{i n t}=\int_{0}^{\infty} d \omega \sum_{k}\left(a_{\omega k}^{\dagger} e^{i \omega t}+a_{\omega k} e^{-i \omega t}\right) J_{k \omega}
$$

Here $\omega$ is the energy of the photons, $k$ represents other degrees of freedom of the photons in addition to their energy (momentum and polarization) and $J_{k \omega}$ are operators acting on the electrons Hilbert space. The photons are taken to be initially in their ground state $a_{\omega k}|0\rangle=0$.

The interaction Hamiltonian eq. (37) is independent of the electron Hamiltonian, ie. it is independent of whether the electron is bound to a proton, a Helium nucleus, a molecule, etc ... . Therefore such a measurement can determine the energy of an unknown state, independently of the Hamiltonian. It can also determine what is the Hamiltonian, since the energy of the emitted photons will differ if the electron is bound to a proton, a Helium nucleus, a molecule, etc .... The price to pay for this universality is that the energy resolution of the measurement and the time necessary for the measurement are constrained by the time-energy uncertainty. The arguments presented in this paper show that this will always be the case for a measurement of energy which does not take into account prior knowledge about the Hamiltonian.

An important limitation of the above measurement scheme is that the emitted photon only reveals the difference in energy between the initial and final state of the atom. If there are several allowed transitions with identical energy differences, then the measurement will not allow these initial states to be differentiated. It would have to be complemented by a second measurement to determine which of the possible final states the atom reached. Nevertheless the important point of this example is to show that in principle it is possible to come close to saturating the time energy uncertainty relation when estimating the energy of a system whose Hamiltonian is unknown.

In order to see how close we come to saturating the time-energy uncertainty in this scheme, let us examine it in more detail. The probability density that a photon is emitted at time $t$ is:

$$
P(\text { decay at time } t)=\gamma e^{-\gamma t} \text {. }
$$

Thus the time it takes to complete the measurement is not well defined. Rather this time is variable but its mean is finite

$$
\int d t t P(\text { decay at time } t)=\gamma^{-1}
$$

This is to be contrasted with the situation envisaged in the previous sections where we required that the measurement be finished after some time interval $\Delta t$.

Let us now consider the energy of the emitted photon. If the true energy of the electronic state is $E_{0}$, then the probability density that the emitted photon has energy $E$ is

$$
P(\text { emitted photon has energy } E)=\frac{1}{\pi} \frac{\gamma}{\gamma^{2}+\left(E-E_{0}\right)^{2}} \text {. }
$$


If we compute the accuracy of the energy measurement using the definition eq. (36), we find

$$
\begin{aligned}
\Delta E & =\int d E P(\text { emitted photon has energy } E)\left|E-E_{0}\right| \\
& =+\infty
\end{aligned}
$$

Thus this measurement satisfies the time energy uncertainty relation. In fact the product $\Delta t \Delta E$ is infinite since both the time it takes to complete the measurement and the energy uncertainty are infinite. However we note that if we modify the definition of $\Delta t$ to be the mean time $\gamma^{-1}$ taken to carry out the measurement, and if we modify the definition of the energy uncertainty $\Delta E$ to be the linewidth $\gamma$, then this measurement does obey a time energy uncertainty relation.

Therefore it may be possible to devise a better energy measurement that saturates the time-energy uncertainty derived in section IV C. Or this uncertainty relation is too strong, and one can prove a weaker form of the uncertainty relation, for instance using as definition of $\Delta t$ and $\Delta E$ the mean time taken to do the measurement and the line width. In the latter case the measurement just described would be optimal in the sense that it would saturate the time energy uncertainty for energy measurements when the Hamiltonian is unknown.

\section{E. Estimating energy when one has partial knowledge about the Hamiltonian}

In the previous section we considered the situation where one wants to estimate the energy of an unknown state but one has no prior knowledge about the Hamiltonian. When some prior knowledge is available the situation is considerably more complicated and the relation between the time used for the measurement and the precision with which the energy can be estimated will depend on the details of the problem.

To illustrate this we consider two examples. First consider the case of two Hamiltonians $H_{1}$ and $H_{2}$ that have the same eigenstates $H_{1} \psi_{k}=E_{1 k} \psi_{k}, H_{2} \psi_{k}=E_{2 k} \psi_{k}$ and their eigenvalues coincide except for one eigenstate: $E_{1 k}=E_{2 k}\left(k \neq k_{0}\right)$ but $E_{1 k_{0}} \neq E_{2 k_{0}}$. Suppose we must determine the energy of an unknown state $\Psi$. A strategy to do this in a short time is to first carry out a von Neumann measurement of the basis $\psi_{k}$ that diagonalizes $H_{1}$ and $H_{2}$ (this can be done in an arbitrarily short time). If one finds that the outcome $k$ is different from $k_{0}$, then one immediately knows the energy. On the other hand if $k=k_{0}$, then to know the energy one must determine what is the Hamiltonian. This takes a time $\Delta t=\pi /\left|E_{1 k_{0}}-E_{2 k_{0}}\right|$. If the unknown state $\Psi$ was uniformly distributed in Hilbert space (denoted $\mathcal{H}$ ), then the probability that $\Psi$ belongs to subspace $k_{0}$ is $1 / \operatorname{dim} \mathcal{H}$ and the average time necessary to determine the energy of the state is $\pi / \operatorname{dim} \mathcal{H}\left|E_{1 k_{0}}-E_{2 k_{0}}\right|$ which is much smaller than the time needed to determine the Hamiltonian. The reason for this difference in time scales is because the particle has most of its support in a part of the Hilbert space where the two Hamiltonians do not differ.

Our second example is superficially similar to the previous one. But more careful consideration show some subtle differences. We consider a particle confined to a (one dimensional) box. The potential in the box vanishes everywhere, except in a corner where it may be either zero or take a large negative value. In order to measure the energy of the particle in a minimum time the following strategy seems natural. First we measure whether the particle is in the corner or not. (This position measurement should be slightly fuzzy so as not to disturb the momentum too much). This measurement can in principle be done in arbitrarily short time. If the particle is not in the corner, we measure its momentum, and hence know its energy. This can also be done in arbitrarily short time. If the particle is in the corner, then we must determine the value of the potential in order to know the energy of the particle. This takes a finite time of order $1 / \Delta V$, where $\Delta V$ is the uncertainty in the potential.

Thus in this case it seems that the minimum time required for the energy measurement depends essentially on the probability of the particle being in the corner of the box rather than on the precision $\Delta E$ with which one wants to know the energy. However the situation is more complicated. The above procedure approximates to some extent a von Neumann measurement of the Hamiltonian operator. Indeed the statistics of the measurement outcomes are such that they reproduce correctly the moments of the Hamiltonian operator. Thus for instance upon repeating the measurement many times one will obtain a good estimate of the average energy $\langle H\rangle$, or the average value of any power of the Hamiltonian $\left\langle H^{n}\right\rangle$. However the above procedure is not equivalent to a von Neumann measurement of the Hamiltonian operator (which is the task we set out to perform). Indeed there are some functions of the Hamiltonian which cannot be estimated correctly with the above procedure.

Suppose for instance that the particle is known to be approximately localized at a distance $d$ from the corner of the box ( $d$ is taken to be much larger than the size of the region where the potential is unknown) and suppose that it is known that the momentum of the particle is approximately $p$. Since the particle is far from the corner, by measuring the momentum of the particle one has some information about its energy. For instance independent repetitions of the measurement will yield estimates of the moments of the Hamiltonian operator $\left\langle H^{n}\right\rangle$. Such measurements of the momentum can be done in an arbitrarily short time. However suppose that one wants to measure the operator $\cos (m d H / p)=\left(e^{i m d H / p}+e^{-i m d H / p}\right) / 2$ (where $m$ is the mass of the particle). This is the real part of the operator that evolves the particle from its initial position up to the corner where the potential is unknown. The expectation value of the above operator clearly depends on the value of the potential in the cor- 
ner, and therefore it can only be determined in a time of order $1 / V$.

The origin of this surprising situation is that in the above example the exact spectrum of $H$ depends on the potential in the corner. When the particle is far from the corner, most questions concerning the energy of the particle are independent of the exact spectrum of $H$. But a variable such as $\cos (m d H / p)$ is sensitive to the exact spectrum and therefore in order to measure it one must know what is the potential in the corner. Operators such as $\cos (m d H / p)$ are called modular variables, and have been introduced in [12].

\section{CONCLUSIONS}

In the present article we have shown that if the Hamiltonian that governs the evolution of a quantum system is unknown, then the time necessary to estimate the Hamiltonian obeys a time-energy uncertainty relation eq. (2). To this end we first gave a simple proof of the problem considered by Childs, Preskill and Renes [5] where there are only two possible Hamiltonians between which one must choose. We then showed how to extend this result to the case where there are many Hamiltonians among which one must choose. The bound we obtained is probably not tight when the unknown Hamiltonians act in a space of large dimensionality $d>2$, and it should be possible to refine it by a more detailed analysis.

Our results concerning the time-energy uncertainty relation applied to estimating Hamiltonians have many applications. In particular they provide new insight about how the time-energy uncertainty applies to energy measurements. It has been shown by Aharonov and Bohm that if the Hamiltonian of the system is known, then the Hamiltonian can in principle be measured in arbitrarily short time. On the other hand we show that if the Hamiltonian is unknown then the energy measurement cannot be done in arbitrarily short time. The minimum amount of time required depends on the details of the problem, for instance what is the prior knowledge about the Hamiltonian, what is the prior knowledge about the state, and exactly what one wants to know about the energy of the state. We show that if one has no prior information about the Hamiltonian, then the time taken to carry out the measurement and the precision with which the energy is measured obey a time-energy uncertainty relation.

We also show that one can devise a measurement of the energy of a quantum system that always works, independently of any prior knowledge about the system. Such a measurement is obtained by coupling the system to an external apparatus that oscillates at all frequencies and such that each frequency is coupled to different degrees of freedom of the apparatus. This is illustrated in eq. (38) in the case where the external apparatus is taken to be the electromagnetic field. Such measurements do not saturate our time energy uncertainty relation, although they do obey a qualitatively similar uncertainty relation between the life time of the state and the line width.

To conclude, we find that the real meaning of energy in quantum mechanics is that of governing the time evolution of a system. To measure the energy one has to determine the time evolution, and this takes time. Thus energy measurements require time, and their precision is limited by the time we have at our disposal. On the other hand, in the examples presented by Aharonov and Bohm [3], the Hamiltonian is known in advance, hence one need not spend time to determine the time-evolution. Instead, one could find out the value of the energy not by determining the time evolution, i.e., not by measuring the actual energy, but by measuring an operator (the operator to which the Hamiltonian is equal) whose numerical eigenvalue is equal to that of the energy. However, we emphasize that although this procedure does yield the numerical values equal to that of the energy, it is not a proper energy measurement. Indeed, if we believe the Hamiltonian to be $H$ but in reality it is different, say $H^{\prime}$, then the value obtained by the instantaneous AharonovBohm measuring procedure (which tells us to measure the operator $H$ ) would no longer be correct, and, furthermore, we would not know that our measurement is wrong. Thus a proper energy measurement necessarily probes the time evolution and therefore cannot be done instantaneously. Rather the time taken to carry out the measurement and the precision with which one knows the energy are constrained by a time energy uncertainty relation $\Delta t \Delta E \geq 1$.

\section{Acknowledgments}

Y.A. acknowledges the support of Grant 471/98 of the Israel Science Foundation established by the Israel Academy of Sciences and Humanities. S.M. is a "research associate" of the Belgium National Research Fund. S.M. and S.P acknowledge funding by project EQUIP (ISTFET program) of the European Union.

\section{APPENDIX A}

In this appendix we show that $D_{0}\left(H_{1}, H_{2}\right)$ defined in eq. (3) is a distance on the space of Hamiltonians. That is $D_{0}$ is 1$)$ positive: $D_{0}\left(H_{1}, H_{2}\right) \geq 0$ with equality if and only if $\left.H_{1}=H_{2} ; 2\right) D_{0}$ is symmetric: $D_{0}\left(H_{1}, H_{2}\right)=D_{0}\left(H_{2}, H_{1}\right)$; and 3$) D_{0}$ obeys the triangle inequality: $D_{0}\left(H_{1}, H_{2}\right) \leq D_{0}\left(H_{1}, H_{3}\right)+D_{0}\left(H_{3}, H_{2}\right)$.

Let us first introduce a norm

$$
\|H\|_{0}=\max \left\{E^{\max }-E^{\min },\left|E^{\max }\right|,\left|E^{\min }\right|\right\}
$$

where $E^{\max }, E^{\min }$ are the largest, smallest eigenvalues of $H$. Now

$$
D_{0}\left(H_{1}, H_{2}\right)=\left\|H_{1}-H_{2}\right\|_{0},
$$


hence if we can prove that $\|H\|_{0}$ is indeed a norm, then it follows immediately that $D_{0}$ is a distance.

We recall that a norm obeys must satisfy the following properties: 1) positivity: $\|H\| \geq 0$ with equality if and only if $H=0 ; 2)$ linearity: $\|\lambda H\|=|\lambda|\|H\|$ for any cnumber $\lambda$; 3) triangle inequality $\left\|H_{1}+H_{2}\right\| \leq\left\|H_{1}\right\|+$ $\left\|H_{2}\right\|$.

Properties 1) and 2) are immediate. Let us consider property 3 . Let us denote by $E_{1}^{\max }$ and $\left|\psi_{1}^{\max }\right\rangle$ the largest eigenvalue of $H_{1}$ and the corresponding eigenvector; by $E_{2}^{\max }$ and $\left|\psi_{2}^{\max }\right\rangle$ the largest eigenvalue of $\mathrm{H}_{2}$ and the corresponding eigenvector; by $E_{12}^{\max }$ and $\left|\psi_{12}^{\max }\right\rangle$ the largest eigenvalue of $H_{1}+H_{2}$ and the corresponding eigenvector. Let us show that $E_{12}^{\max } \leq E_{1}^{\max }+E_{2}^{\max }$.

[1] L. D. Landau and R. Peierls, Z. Physik 69 (1931) 56

[2] L. Landau and E. Lifschitz, Quantum Mechanics (Pergamon Press, New York, 1958), pp. 150-153

[3] Y. Aharonov and D. Bohm, Phys. Rev. 122 (1961) 1649

[4] Y. Aharonov and B. Reznik, Phys. Rev. Lett. 84 (2000) 1368

[5] A. M. Childs, J. Preskill, J. Renes, J. Mod. Optics, 47 (2000) 155

[6] G. Mitchison and S. Massar, Phys, Rev. A 63 (2001), 032105

[7] C. H. Bennett, E. Bernstein, G. Brassard, and U. V. Vazirani, SIAM Journal on Computing 26, 1510 (1997), quant-ph/9701001
We have

$$
\begin{aligned}
E_{12}^{\max } & =\left\langle\psi_{12}^{\max }\left|\left(H_{1}+H_{2}\right)\right| \psi_{12}^{\max }\right\rangle \\
& =\left\langle\psi_{12}^{\max }\left|H_{1}\right| \psi_{12}^{\max }\right\rangle+\left\langle\psi_{12}^{\max }\left|H_{2}\right| \psi_{12}^{\max }\right\rangle \\
& \leq\left\langle\psi_{1}^{\max }\left|H_{1}\right| \psi_{1}^{\max }\right\rangle+\left\langle\psi_{2}^{\text {max }}\left|H_{2}\right| \psi_{2}^{\text {max }}\right\rangle \\
& =E_{1}^{\max }+E_{2}^{\max } .
\end{aligned}
$$

Similarly we have $E_{12}^{\text {min }} \geq E_{1}^{\text {min }}+E_{2}^{\text {min }}$ where $E_{12}^{\text {min }}, E_{1}^{\min }, E_{2}^{\min }$ are the smallest eigenvalues of $H_{1}+$ $H_{2}, H_{1}, H_{2}$ respectively. The triangle inequality follows from these relations between eigenvalues and from the definition of $\|H\|_{0}$.

[8] E. Farhi and S. Gutmann, Phys. Rev. A 57, 2403 (1998), quant-ph/9612026

[9] L. K. Grover, A fast quantum mechanical algorithm for database search, in Proc. 28th Annual ACM Symposium on the Theory of Computing, 212 (ACM Press, New York, 1996), quant-ph/9605043

[10] C. W. Helstrom, Quantum Detection and Estimation Theory, (Academic Press, New York,1976)

[11] D. Bruss, C. Macchiavello, Phys.Lett. A253 (1999) 249251

[12] Y. Aharonov, H. Pendleton and A. Petersen, Int. Jour. of Theor. Physics, Vol.2, No.3, 213 (1969) 